\title{
Mineralização de esterco de ovinos e sua influência na produção de alface
}

\author{
Cícero Célio de Figueiredo; Maria Lucrécia G Ramos; Concepta M McManus; Adriana M de Menezes \\ UnB-FAV, C. Postal 04508, 70910-970 Brasília-DF; cicerocf@unb.br
}

\section{RESUMO}

Diversos resíduos orgânicos são utilizados na agricultura sem o adequado conhecimento da sua dinâmica de mineralização. Avaliou-se a mineralização de esterco de ovinos e sua influência na produção de alface. Utilizou-se o delineamento experimental de blocos ao acaso com três repetições. Foram utilizadas $25 \mathrm{t} \mathrm{ha}^{-1}$ como dose de esterco para cada um dos seguintes tratamentos: 1) esterco de ovinos que se alimentaram de feno de mandioca (PAM); 2) esterco de ovinos que se alimentaram de subproduto de ervilha (ERV); 3) esterco de ovinos que se alimentaram de feno de capim coast-cross (FCC); 4) esterco de ovinos que se alimentaram de subproduto de saccharina (SAC) e 5) solo sem aplicação de esterco (testemunha). Foi determinada semanalmente a respiração basal do solo, utilizada como indicador de mineralização da matéria orgânica. A massa fresca de alface foi avaliada como medida de produção. Os tratamentos ERV, FCC e SAC apresentaram ganhos de massa fresca na ordem de 68, 65 e $62 \%$ em relação à testemunha e de 43,39 e $33 \%$ em relação ao PAM, respectivamente. A produção menor promovida pelo PAM, em relação às demais, pode ser explicada pela forma de mineralização da matéria orgânica que apresentou elevada respiração microbiana cinco dias após o transplantio, com acentuado declínio, nas medições subseqüentes, ao longo do ciclo da cultura. Os demais tratamentos apresentaram mineralização sincronizada com conseqüente aumento na produção de massa fresca.

Palavras-chave: Lactuca sativa, respiração microbiana, esterco, adubação orgânica.

\begin{abstract}
Mineralization of sheep manure and its influence on lettuce production

Several organic wastes are used in agriculture with no precise knowledge about the mineralization dynamics of these materials. In this study the sheep manure mineralization and its influence on the lettuce production was evaluated. A randomized block design with three replications was used. Five treatments were studied using $25 \mathrm{t}$ ha $^{-1}$ as dose of manure: 1) sheep manure obtained from animals fed with cassava straw (PAM); 2) sheep manure obtained from animals fed with residue of pea crop (ERV); 3) sheep manure obtained from animals fed with Coast-Cross hay (FCC), 4) sheep manure obtained from animals fed with saccharin residue (SAC) and 5) soil without application of manure (control). Weekly the basal respiration was determined and used as an indicator of organic matter mineralization. Lettuce fresh mass was evaluated as a measure of production. Treatments ERV, FCC and SAC showed superior weight gains of 68, 65 and $62 \%$ compared to the control and 43,39 and 33\% compared to MAP, respectively. Lower production promoted by the MAP in relation to the other treatments can be explained by organic matter mineralization that showed a high microbial respiration five days after transplanting, with marked decline in subsequent measurements during the crop cycle. The other systems showed mineralization synchronized with the production increase of lettuce fresh mass.
\end{abstract}

Keywords: Lactuca sativa, microbial respiration, manure, organic fertilization.

\section{(Recebido para publicação em 21 de junho de 2010; aceito em 15 de fevereiro de 2012)} (Received on June 21, 2010; accepted on February 15, 2012)

A alface (Lactuca sativa) está entre as hortaliças folhosas mais consumidas no mundo (Gomes et al., 2008). Esta cultura possui baixo valor calórico e é fonte de vitaminas e sais minerais (Oshe et al., 2001), bem como apresenta grande potencial de produção com adubos orgânicos (Moraes et al., 2006).

Com o uso intensivo de adubos minerais e agrotóxicos, as altas produtividades alcançadas têm sido questionadas nos últimos anos, não só pelas contradições econômicas e ecológicas, mas também por desprezar aspectos qualitativos importantes da produção (Santos et al., 1994).

Os adubos orgânicos aplicados ao solo representam uma alternativa para aumentar a produção das culturas. En- tretanto, dependendo de sua composição química, taxa de mineralização e teor de nitrogênio, que por sua vez sofrem influências das condições climáticas, os adubos orgânicos em doses elevadas tornam-se prejudiciais às culturas.

Com a adição ao solo de um material rico em carbono orgânico, como o esterco de ovinos, parte deste é utilizada pelos microrganismos como fonte de energia, o que promove aumento na atividade microbiológica e conseqüente liberação de $\mathrm{CO}_{2}$. A respiração microbiana reflete a atividade microbiológica do solo e é medida pela quantificação de $\mathrm{CO}_{2}$ liberado e/ou de $\mathrm{O}_{2}$ absorvido, resultante da atividade dos microrganismos (Paul \& Clark, 1989).

A medição da respiração microbia- na é uma forma de estimar a atividade dos microrganismos do solo, a qual reflete a velocidade de decomposição da matéria orgânica do solo ou de algum material a ele adicionado. Quando um material orgânico é adicionado ao solo, os microrganismos realizam sua decomposição, a qual pode ocorrer de forma rápida se houver fatores propícios como umidade, $\mathrm{pH}$, temperatura, mas principalmente nutrientes e cadeias de carbono (fonte de energia). A ocorrência de alta atividade microbiana indica que a decomposição do material adicionado é rápida e os nutrientes são mineralizados e disponibilizados para as plantas em menor tempo, o que muitas vezes é uma característica buscada em um adubo orgânico (Severino et al., 2004). 
Diversos fatores interferem na decomposição e mineralização dos resíduos orgânicos, dentre estes, pode-se citar a relação $\mathrm{C}: \mathrm{N}$ dos estercos, características físico-químicas e biológicas, além da temperatura e da umidade do solo. Além desses fatores, outras características podem influenciar o processo de decomposição desses resíduos. O esterco bovino, por exemplo, apresenta relação $\mathrm{C} / \mathrm{N}$ maior que o esterco de caprinos e ovinos. Apesar disso, em vários estudos já realizados, apresenta maior taxa de decomposição, o que pode ser atribuído à sua estrutura que facilita a ação de microrganismos. Já os estercos de caprinos e ovinos, por possuírem uma espécie de membrana que os revestem e tornam-os duros quando excretados, possuem uma maior resistência à decomposição (Petersen et al., 1998).

Em estudo com aplicação de diversos estercos, Brito et al. (2005) concluíram que o esterco ovino foi o resíduo que determinou as principais alterações das propriedades químicas do solo, uma vez que em relação à testemunha, promoveu os maiores aumentos de cálcio, matéria orgânica e capacidade de troca de cátions. Esses efeitos, porém, só foram intensificados a partir do terceiro mês após a aplicação. A velocidade de decomposição e consequente mineralização dos resíduos orgânicos interferem diretamente na disponibilidade de nutrientes para as plantas, principalmente para aquelas de ciclo curto, como a alface.

Há carência de informações sobre a forma de mineralização de estercos de ovinos submetidos a diferentes fontes de alimentação, bem como, sobre a produção de alface submetida a fontes orgânicas diferenciadas. Este trabalho teve por objetivo estudar a mineralização de estercos de ovinos submetidos a fontes de alimentos diferenciadas e sua influência na produção de alface.

\section{MATERIAL E MÉTODOS}

O trabalho foi conduzido no Centro de Manejo de Ovinos da Universidade de Brasília (UnB), localizada em Vargem Bonita, Brasília (DF) $\left(15^{\circ} 55^{\prime} 58^{\prime}\right.$ S e 4751'02' 'W, altitude de $1000 \mathrm{~m}$ ). Foram utilizados estercos de ovinos submetidos a diferentes dietas em experimento zootécnico, realizado no setor de ovinocultura da UnB.

Os estercos foram obtidos de animais submetidos às diferentes dietas isoprotéicas e isoenergéticas. Os animais foram criados individualmente em baias, com água e sal mineral à vontade. Aos animais foram fornecidas diversas fontes alimentares, apresentando $40 \%$ de concentrado e $60 \%$ composto pelos seguintes materiais: FCC (feno de coast-cross (Cynodon Dactylon)); ERV (subproduto da lavoura de ervilha desidratada (Pisum sativum)); PAM (feno da parte aérea da mandioca (Manihot esculenta)); SAC (saccharina (Saccharum ssp.)). O concentrado foi composto por mistura de farelo de soja (S) e milho (M), nas proporções: $\mathrm{FCC}=11,24 \%$ de $\mathrm{M}$ e $28,76 \%$ de $\mathrm{S} ; \mathrm{ERV}=24,10 \%$ de $\mathrm{M}$ e $15,90 \%$ de $\mathrm{S} ; \mathrm{PAM}=19,08 \%$ de $\mathrm{M}$ e $20,92 \%$ de $\mathrm{S} ; \mathrm{SAC}=16,59 \%$ de $\mathrm{M}$ e $23,48 \%$ de $\mathrm{S}$.

O ERV foi composto por um subproduto desidratado da lavoura de ervilha com tamanho de partícula de $1,5 \mathrm{~cm}$, obtido em uma agroindústria localizada no entorno do Distrito Federal. O feno de capim coast-cross teve sua procedência também na região do entorno do Distrito Federal. Este material foi fornecido aos animais após ser triturado em partículas de $4 \mathrm{~cm}$. Caules, pecíolos e folhas de mandioca foram usados para a confecção do feno (PAM), após secagem e trituração, partículas de 2 $\mathrm{cm}$ foram utilizadas. Saccharina foi confeccionada com colmo e folhas da cana-de-açúcar (Saccharum ssp.). A cana-de-açúcar foi cortada e mantida por 24 horas em local coberto. Após este período, o material foi triturado em partículas de $4 \mathrm{~cm}$, adicionado $5 \%$ de uréia pecuária e revolvido por 17 dias até a secagem total.

Os estercos foram coletados diariamente por 45 dias e foi feita uma mistura de esterco dos animais do mesmo tratamento alimentar. Após isso, foi instalado um experimento de campo.

A análise do solo, classificado como Latossolo Vermelho, apresentou a composição química: $\mathrm{pH}\left(\mathrm{H}_{2} \mathrm{O}\right)=6,3 ; \mathrm{P}=$ $19,5 \mathrm{mg} \mathrm{dm}^{-3} ; \mathrm{K}^{+}=80,6 \mathrm{mg} \mathrm{dm}{ }^{-3} ; \mathrm{Al}^{+3}=$ $0,0 \mathrm{cmol}_{\mathrm{c}} \mathrm{dm}^{-3} ; \mathrm{H}+\mathrm{Al}=0,0 \mathrm{cmol}_{\mathrm{c}} \mathrm{dm}^{-3}$;
$\mathrm{Ca}^{2+}+\mathrm{Mg}^{+2}=4,3 \mathrm{cmol}_{\mathrm{c}} \mathrm{dm}^{-3}$; matéria orgânica total $=46 \mathrm{~g} \mathrm{~kg}^{-1}$. As determinações foram realizadas conforme Embrapa (1999). Para a determinação do fósforo e do potássio utilizou-se o extrator Mehlich-1. Os cátions trocáveis $\mathrm{Ca}^{2+}, \mathrm{Mg}^{2+}$ e $\mathrm{Al}^{3+}$ foram extraídos com $\mathrm{KCl} 1 \mathrm{~mol} \mathrm{~L}^{-1}$. A acidez potencial $(\mathrm{H}+$ Al) foi determinada após a extração com acetato de cálcio com pH igual a 7,0. A matéria orgânica foi determinada por oxidação via úmida com dicromato de potássio, sem aquecimento externo. As características químicas dos estercos são apresentadas na Tabela 1 .

Utilizou-se o delineamento experimental em blocos ao acaso com três repetições, com parcelas de $2 \mathrm{~m}^{2}$. Cinco tratamentos foram avaliados, sendo $25 \mathrm{t}$ ha $^{-1}$ a dose de esterco aplicada: 1) esterco de ovinos que se alimentaram de feno de mandioca (PAM); 2) esterco de ovinos que se alimentaram de subproduto de ervilha (ERV); 3) esterco de ovinos que se alimentaram de feno de capim coast-cross (FCC); 4) esterco de ovinos que se alimentaram de subproduto de saccharina (SAC) e 5) solo sem aplicação de esterco (testemunha).

As sementes de alface, cultivar Mauren, do tipo americana, foram semeadas em bandejas de isopor contendo substrato comercial e as mudas transplantadas quando apresentavam três folhas abertas. $\mathrm{O}$ espaçamento adotado foi de $25 \times 25 \mathrm{~cm}$, totalizando 40 plantas por parcela. Como tratos culturais foram realizadas irrigações diárias por aspersão, de forma a manter o teor de umidade no solo próximo à capacidade de campo. Também foram realizadas capinas manuais.

Semanalmente foram coletadas amostras do solo na profundidade de 0 a $10 \mathrm{~cm}$. Foi determinada a respiração basal, de acordo com a metodologia de Alef \& Nannipieri (1995), como indicadora da mineralização da matéria orgânica. As amostras foram tamisadas em peneiras com abertura de $8 \mathrm{~mm}$, retirando-se fragmentos de raízes e restos vegetais. Os teores de umidade das amostras foram corrigidos para $80 \%$ da capacidade máxima de retenção de água no solo. As amostras foram divididas em subamostras (triplicatas) de $20 \mathrm{~g}$ de solo e colocadas no interior de vidros 
herméticos de $500 \mathrm{~mL}$, juntamente com um frasco de vidro contendo $10 \mathrm{~mL}$ de $\mathrm{KOH} 0,3 \mathrm{~mol} \mathrm{~L}^{-1}$. As amostras foram incubadas por sete dias. Posteriormente, para quantificar o $\mathrm{CO}_{2}$ liberado durante a incubação, os frascos contendo o $\mathrm{KOH}$ $0,3 \mathrm{~mol} \mathrm{~L}^{-1}$, na presença de $3 \mathrm{~mL}$ de $\mathrm{BaCl}_{2}$ 0,082 $\mathrm{mol} \mathrm{L}^{-1}$ foram titulados com $\mathrm{HCl} 0,1 \mathrm{~mol} \mathrm{~L}^{-1}$ em frascos de $100 \mathrm{~mL}$, contendo duas gotas de fenolftaleína. A quantidade de $\mathrm{CO}_{2}$ liberado foi calculada pelo número de moles de $\mathrm{KOH}$ iniciais menos o número de moles de $\mathrm{KOH}$ que reagiu com o $\mathrm{HCl}$ 0,1 $\mathrm{mol} \mathrm{L}^{-1}$.

Uma amostra composta por quatro plantas da área útil da parcela $\left(0,8 \mathrm{~m}^{2}\right)$, escolhida aleatoriamente, colhida quarenta e cinco dias após o transplantio, foi usada para determinação dos valores da massa fresca (MF) total, em g/planta.

Os dados foram submetidos à análise de variância e as médias comparadas pelo teste de Duncan $(\mathrm{p}<0,05)$, para determinar o efeito dos tratamentos sobre a respiração microbiana e a produção de alface. O efeito do tempo após o transplantio na respiração basal foi avaliado através de análise de regressão.

\section{RESULTADOS E DISCUSSÃO}

Influência sobre a produção de alface - Os valores de matéria fresca da parte aérea aos $45 \mathrm{~d}$ após plantio variaram de $159 \mathrm{~g} /$ planta no solo sem adição de esterco de ovinos a $494 \mathrm{~g} /$ planta naquele que recebeu esterco de ovinos alimentados com subproduto de ervilha (ERV) (Tabela 2). Esses valores são semelhantes aos encontrados em trabalhos com a utilização de adubação orgânica ou organomineral em alface (Santos et al., 1994; Yuri et al., 2004; Figueiredo \& Ramos, 2009). A dose aplicada de $25 \mathrm{t} \mathrm{ha}^{-1}$ de esterco ovino promoveu produção de massa fresca total da parte aérea de alface similar àquela que recebeu $50 \mathrm{tha}^{-1}$ de esterco bovino, encontrada por Figueiredo \& Ramos (2009). As produções obtidas neste trabalho são similares àquelas obtidas por Paula et al. (2009), quando a alface recebeu $25 \mathrm{t} \mathrm{ha}^{-1}$ de esterco bovino aplicado em pré-plantio e $250 \mathrm{~g} \mathrm{~m}^{-2}$ de cama de aviário em cobertura.

Tabela 1. Caracterização química de estercos de ovinos submetidos a diferentes dietas alimentares (chemical characterization of sheep manure subjected to different diets). Brasília, UnB, 2010.

\begin{tabular}{|c|c|c|c|c|c|c|c|c|c|c|}
\hline \multirow[t]{2}{*}{ Esterco } & \multirow[t]{2}{*}{ pH } & \multirow[t]{2}{*}{$\begin{array}{c}\text { Umidade } \\
\left(65^{\circ} \mathrm{C}\right)\end{array}$} & $\begin{array}{l}\text { Carbono } \\
\text { orgânico }\end{array}$ & $\begin{array}{c}N \\
\text { total }\end{array}$ & $\begin{array}{c}\text { Relação } \\
\text { C:N }\end{array}$ & $\begin{array}{l}\mathrm{P}_{2} \mathrm{O}_{5} \\
\text { total }\end{array}$ & $\mathbf{K}_{2} \mathbf{O}$ & $\mathbf{S}$ & \multirow[t]{2}{*}{ Ca } & \multirow[t]{2}{*}{ Mg } \\
\hline & & & \multicolumn{6}{|c|}{$\%$} & & \\
\hline ERV & 7,9 & 8,7 & 34,7 & 1,80 & 19 & 1,36 & 2,24 & 0,23 & 0,9 & 0,4 \\
\hline PAM & 7,5 & 8,9 & 36,5 & 1,27 & 29 & 0,71 & 1,45 & 0,17 & 0,8 & 0,3 \\
\hline FCC & 7,5 & 8,9 & 35,9 & 1,21 & 28 & 1,05 & 1,47 & 0,25 & 0,6 & 0,3 \\
\hline SAC & 7,5 & 9,8 & 36,0 & 1,66 & 22 & 1,18 & 1,53 & 0,23 & 0,7 & 0,3 \\
\hline
\end{tabular}

$\mathrm{ERV}=$ esterco produzido por ovinos que se alimentaram de $60 \%$ do subproduto da lavoura de ervilha desidratada; PAM= esterco produzido por ovinos que se alimentaram de $60 \%$ feno da parte aérea da mandioca; $\mathrm{FCC}=$ esterco produzido por ovinos que se alimentaram de $60 \%$ de feno de coast-cross; $\mathrm{SAC}=$ esterco produzido por ovinos que se alimentaram de $60 \%$ de saccharina; Todas as dietas receberam mais $40 \%$ de concentrado (ERV $=$ manure produced by sheeps fed with a byproduct of the dried pea crop; PAM= manure produced by sheeps fed with hay of cassava foliage; $\mathrm{FCC}=$ manure produced by sheeps fed with $60 \%$ hay of coast-cross; $\mathrm{SAC}=$ manure produced by sheeps fed with $60 \%$ of saccharina; All diets received $40 \%$ of concentrate).

As plantas fertilizadas com PAM, ERV, FCC e SAC apresentaram ganhos de massa fresca na ordem de 43, 68, 65 e $62 \%$ respectivamente, em relação à testemunha. Em relação ao tratamento PAM, os ganhos de massa fresca de alface foram de 43, 39 e 33\% com ERV, FCC e SAC, respectivamente. Esses resultados demonstram que a dieta alimentar diferenciada promoveu estercos com potenciais de mineralização diferenciados. Os baixos teores de fósforo e enxofre do PAM, em relação aos demais, pode ser a justificativa da menor produção de massa fresca promovida pelo PAM.

Em trabalho com vermicompostos provenientes de diferentes resíduos orgânicos usados como substratos, Castilhos et al. (2007) encontraram que o vermicomposto que utilizou o esterco ovino como substrato promoveu maior matéria seca em plantas de alface quando comparado com outros resíduos como esterco bovino, borra de café e erva mate.

Mineralização dos resíduos orgânicos - Na Figura 1 são apresentadas as dinâmicas da atividade microbiana do solo, após a aplicação dos diversos estercos de ovinos. Verifica-se que, em relação à testemunha, todas as aplicações de estercos aumentaram a quantidade de matéria orgânica mineralizada, medida
Tabela 2. Massa fresca da parte aérea da planta de alface (g/planta) produzida com estercos de ovinos (fresh shoot mass of lettuce plants (g/plant), produced with sheep manure). Brasília, UnB, 2010.

\begin{tabular}{lc}
\hline Tratamento $^{1}$ & Massa fresca \\
\hline TEST & $159 \mathrm{c}^{2}$ \\
PAM & $281 \mathrm{~b}$ \\
ERV & $494 \mathrm{a}$ \\
FCC & $465 \mathrm{a}$ \\
SAC & $424 \mathrm{a}$ \\
DMS & 71 \\
\hline CV (\%) & 9 \\
\hline
\end{tabular}

${ }^{1}$ TEST $=$ solo sem adição de esterco de ovinos; os demais tratamentos receberam $25 \mathrm{t} \mathrm{ha}^{-1}$ de esterco de ovinos que se alimentaram de feno de mandioca (PAM); esterco de ovinos que se alimentaram de ervilha (ERV); esterco de ovinos que se alimentaram de feno de capim coast-cross (FCC); esterco de ovinos que se alimentaram de saccharina (SAC); ${ }^{2}$ valores seguidos da mesma letra na coluna não apresentam diferença significativa, entre si, pelo teste de Duncan a $5 \%$ de significância $(\mathrm{p}<0,05)$ $\left({ }^{1} \mathrm{TEST}=\right.$ soil without ovine manure; the other treatments received $25 \mathrm{tha}^{-1}$ of manure of sheeps fed with hay of cassava (PAM); manure of sheeps fed with dried pea (ERV); manure of sheep fed with hay of coast-cross grass (FCC); manure of sheeps fed with hay of saccharina (SAC); ${ }^{2}$ values followed by same letter in column are not significantly different between themselves by Duncan test at $5 \%$ of significance $(\mathrm{p}<0,05))$. 


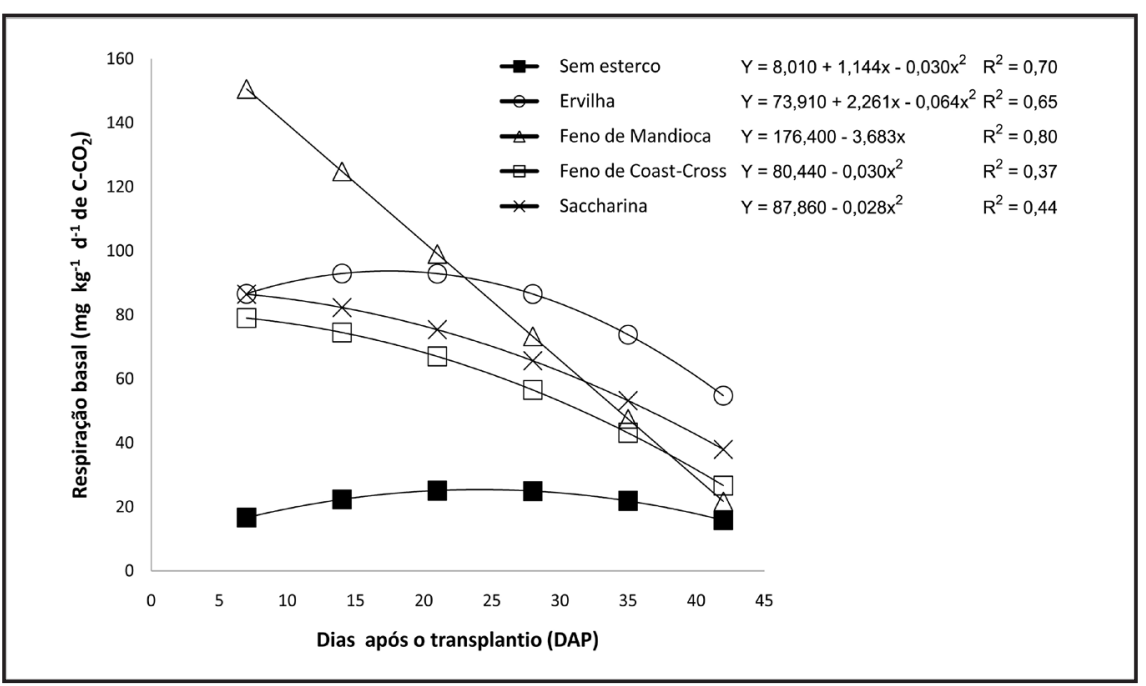

Figura 1. Mineralização de resíduos orgânicos (estercos de ovinos) medida pela respiração basal (mineralization of organic waste (sheep manure) measured by basal respiration). Brasília, UnB, 2010.

Tabela 3. Mineralização da matéria orgânica medida pela respiração basal (mg/kg/dia de $\mathrm{C}-\mathrm{CO}_{2}$ ) em diferentes épocas, durante o ciclo da alface (mineralization of organic matter as measured by basal respiration $\left(\mathrm{mg} / \mathrm{kg} /\right.$ day of $\left.\mathrm{C}-\mathrm{CO}_{2}\right)$ at different times during the lettuce cycle). Brasília, UnB, 2010.

\begin{tabular}{|c|c|c|c|c|c|c|}
\hline \multirow{2}{*}{ Tratamento } & \multicolumn{6}{|c|}{ Época (DAP) ${ }^{1}$} \\
\hline & 7 & 14 & 21 & 28 & 35 & 42 \\
\hline TEST $^{2}$ & $13,6 \mathrm{~d}$ & $19,3 \mathrm{~d}$ & $22,9 \mathrm{c}$ & $11,1 \mathrm{c}$ & $12,5 \mathrm{~b}$ & $13,9 \mathrm{~b}$ \\
\hline ERV & 84,1 b & $101,6 \mathrm{~b}$ & $77,2 \mathrm{~b}$ & 89,2 a & 77,9 a & 52,6 a \\
\hline PAM & $163,1 \mathrm{a}$ & $123,5 \mathrm{a}$ & $63,3 \mathrm{~b}$ & 80,3 a & 52,4 a & $28,1 \mathrm{ab}$ \\
\hline FCC & $68,0 \mathrm{c}$ & $115,2 \mathrm{ab}$ & $36,5 \mathrm{c}$ & $67,2 \mathrm{~b}$ & 51,9 a & $25,0 a b$ \\
\hline SAC & $87,3 \mathrm{~b}$ & $65,2 \mathrm{c}$ & 97,1 a & $70,0 \mathrm{~b}$ & 76,5 a & $23,2 \mathrm{~b}$ \\
\hline DMS & 8,4 & 12,8 & 21,1 & 8,9 & 30,1 & 28,7 \\
\hline CV (\%) & 4,7 & 7,0 & 17,5 & 5,2 & 27,2 & 33,7 \\
\hline
\end{tabular}

${ }^{1} \mathrm{DAP}=$ dias após o transplantio; ${ }^{2} \mathrm{TEST}=$ solo sem adição de esterco de ovinos; os demais tratamentos receberam $25 \mathrm{tha}^{-1}$ de esterco de ovinos que se alimentaram de feno de mandioca (PAM); esterco de ovinos que se alimentaram de ervilha (ERV); esterco de ovinos que se alimentaram de feno de capim coast-cross (FCC); esterco de ovinos que se alimentaram de saccharina (SAC); ${ }^{2}$ valores seguidos da mesma letra na coluna não apresentam diferença significativa, entre si, pelo teste de Duncan a $5 \%$ de significância $(\mathrm{p}<0,05)\left({ }^{1} \mathrm{DAP}=\right.$ days after transplanting; ${ }^{2}$ TEST $=$ soil without ovine manure; other treatments received $25 \mathrm{tha}^{-1}$ of manure of sheeps fed with hay of cassava (PAM); manure of sheeps fed with dried pea (ERV); manure of sheeps fed with hay of coast-cross grass (FCC); manure of sheeps fed with hay of saccharina (SAC); ${ }^{2}$ values followed by same letter in column are not significantly different between themselves by Duncan test at $5 \%$ of significance $(p<0,05))$.

pela evolução de $\mathrm{C}-\mathrm{CO}_{2}$, chamada de respiração basal do solo.

De maneira geral, todos os estercos promoveram elevada taxa de atividade microbiana inicial, logo após a aplicação ao solo, apresentando tendência de queda da respiração com o passar do tempo, mas com intensidades diferenciadas. $\mathrm{O}$ esterco de animais que receberam alimentação com feno da mandioca (PAM) após a sua aplicação ao solo.

No caso do esterco PAM, a rápida liberação de nutrientes justifica a menor produção de massa fresca de plantas de alface proporcionada produzida em relação aos demais estercos utilizados, ainda que tenha superado o tratamento testemunha, que não recebeu esterco algum. Nos demais tratamentos, devido à forma de mineralização mais sincronizada, os estercos promoveram maiores massas de alface.

Silva et al. (2007) encontraram diferenças na taxa de mineralização da matéria orgânica após a aplicação de dois biofertilizantes diferentes. Os autores justificaram que o biofertilizante puro favoreceu a maior atividade microbiana, o que se refletiu em maior mineralização da matéria orgânica. Esses resultados reforçam que pequenas diferenças na composição química dos resíduos utilizados promovem distintas dinâmicas de mineralização.

$\mathrm{Na}$ ausência de plantas, Severino et al. (2004) verificaram que a torta de mamona aplicada ao solo proporcionou elevada taxa de atividade microbiana, crescente ao longo dos trinta dias de incubação. Ao contrário, esterco e bagaço de cana proporcionaram pequena e constante atividade microbiana ao longo do tempo. Os autores justificaram que a elevada mineralização da torta de mamona foi decorrente da grande quantidade de nitrogênio presente nesse resíduo. A ocorrência de alta atividade microbiana indica que a decomposição do material adicionado é rápida e os nutrientes são mineralizados e disponibilizados para as plantas em menor tempo, o que muitas vezes é uma característica desejável em um adubo orgânico (Severino et al., 2004).

Na Tabela 3, são apresentadas as taxas de mineralização dos resíduos orgânicos, medidas pela respiração basal de microrganismos, nas diferentes épocas de coleta, ao longo do ciclo da alface. Os valores variaram de 11,1 aos 28 DAP no solo que não recebeu aplicação de esterco de ovinos a $163,1 \mathrm{mg} / \mathrm{kg} / \mathrm{dia}$ de $\mathrm{C}-\mathrm{CO}_{2}$ no PAM aos 7 DAP. Silva et al. (2007) encontraram variação de 25 a 45 $\mathrm{mg} / \mathrm{kg} /$ dia de $\mathrm{C}-\mathrm{CO}_{2}$, dependendo da dose e do tipo de biofertilizante aplicado durante o ciclo do milho. 
Logo após a aplicação ao solo, aos 7 DAP, o PAM promoveu elevada respiração microbiana, superior aos demais tratamentos. A partir dos 35 DAP, não houve diferença nos valores de mineralização entre os diferentes estercos. O solo que não recebeu esterco sempre apresentou os menores valores de respiração em todas as épocas avaliadas.

Conclui-se, de maneira geral, que a adição de esterco de ovino promove aumento da produção de alface e da atividade microbiana do solo. Estercos de ovinos submetidos a diferentes dietas promovem diferentemente a produção de alface.

O esterco oriundo de ovinos que receberam alimentação com feno de mandioca apresenta rápida mineralização inicial e decréscimo acentuado da respiração do solo, com consequente diminuição da produção de alface, em relação aos demais estercos utilizados, mas superior ao solo que não recebeu aplicação de esterco.

\section{AGRADECIMENTOS}

Os autores agradecem à Fundação de Apoio à Pesquisa do Distrito Federal, à CAPES, CNPq e ao Decanato de Pesquisa e Pós-graduação da Univesidade de Brasília pelo auxílio financeiro que possibilitou o desenvolvimento desse trabalho.

\section{REFERÊNCIAS}

ALEF K; NANNIPIERI P. 1995. Methods in applied soil microbiology and biochemistry. San Diego, CA: Academic Press. 576p.

BRITO OR; VENDRAME PRS; BRITO RM. 2005. Alterações das propriedades químicas de um Latossolo Vermelho distroférrico submetido a tratamentos com resíduos orgânicos. Semina: Ciências Agrárias 26: 33-40.

CASTILHOS DD; SOUZA LM; MORSELLI TBGA; CASTILHOS RMV. 2007. Alterações químicas no solo e produção de alface decorrentes da adição de vermicompostos. Magistra 19: 143-149.

EMBRAPA. Centro Nacional de Pesquisa de Solos. 1999. Manual de análises químicas de solos, plantas e fertilizantes. Campinas: Embrapa Informática Agropecuária; Rio de Janeiro: Embrapa Solos. 370p.

FIGUEIREDO CC; RAMOS MLG. 2009. Biomassa microbiana do solo e produção de alface em função da dose de $\mathrm{N}$ e adubo orgânico. Bioscience Journal 25: 9-15.

GOMES LAA; RODRIGUES AC; COLLIER LS; FEITOSA SS. 2008. Produção de mudas de alface em substrato alternativo com adubação. Horticultura Brasileira 26: 359-363.

MORAES SRG; CAMPOS VP; POZZA EA; FONTANETTI A; CARVALHO GJ; MAXIMINIANO C. 2006. Influência de leguminosas no controle de fitonematóides em cultivo orgânico de alface americana e repolho.
Fitopatologia Brasileira 31: 188-191.

OSHE S; DOURADO-NETO D; MANFRON PA; SANTOS OS. 2001. Qualidade de cultivares de alface produzidos em hidroponia. Scientia Agrícola 58: 181-185.

PAULA PD; GUERRA JGM; RIBEIRO RLD I; CESAR MNZ; GUEDES RE; POLIDORO JC. 2009. Viabilidade agronômica de consórcios entre cebola e alface no sistema orgânico de produção. Horticultura Brasileira 27: 202-206.

PAUL EA; CLARK FE. 1989. Soil microbiology and biochemistry. San Diego, CA: Academic Press. 275p.

PETERSEN SO; LIND AM; SOMMER SG. 1998. Nitrogen and organic matter losses during storage of cattle and pig manure. Journal of Agricultural Science 130: 69-79.

SANTOS RHS; CASALI VWD; CONDE AR; MIRANDA LCG. 1994. Qualidade de alface cultivada em composto orgânico. Horticultura Brasileira 12: 29-32.

SEVERINO LS; COSTA FX; BELTRÃO NEM; LUCENA MA; GUIMARÃES MMB. 2004. Mineralização da torta de mamona, esterco bovino e bagaço de cana estimada pela respiração microbiana. Revista de Biologia e Ciências da Terra 5: 20-26.

SILVA AP; SILVEIRA JPA; SANTOS FRAGA VS; SILVA E; SOUZA JM; LIMA LPF; NASCIMENTO J; MEDEIROS A. 2007. Respiração edáfica após aplicação de biofertilizantes em cultivo orgânico de milho. Revista Brasileira de Agroecologia 2: 12511254.

YURI JE; RESENDE GM; RODRIGUES JÚNIOR JC; MOTA JH; SOUZA RJ. 2004. Efeito de composto orgânico sobre a produção e características comerciais de alface americana. Horticultura Brasileira 22: 127-130. 